Trauma Berufskrankh 2017 · 19 (Suppl 3):S340-S346 https://doi.org/10.1007/s10039-017-0319-x Online publiziert: 19. Oktober 2017

(c) Springer Medizin Verlag GmbH 2017

CrossMark

Traumatische Nervenverletzungen im Zusammenhang mit Unfallereignissen oder im Zusammenhang mit ärztlichen Eingriffen sind zwar eher selten, ihre Inzidenz wird mit 1,6-2 \% und die Prävalenz mit $5 \%$ angegeben, aber für den Betroffenen sind sie aufgrund der resultierenden Ausfälle mit Lähmungen, Sensibilitätsstörungen und gelegentlich auch durch die Entwicklung neuropathischer Schmerzen von einiger Relevanz.

Durch ihren z.T. sehr oberflächlichen Verlauf oder die enge anatomische Beziehung zu den langen Röhrenknochen werden Nerven häufig durch direkte Gewalteinwirkung bei Frakturen und Gelenkluxationen verletzt oder auch sekundär im Rahmen der operativen Unfallversorgung geschädigt, wie z. B. eine Nervenkompression im Rahmen der osteosynthetischen Versorgung durch Kompression, Hakenzug, Durchtrennung oder ungünstige Lagerung [1]. Das Vermeiden derartiger Läsionen im Rahmen der operativen Versorgung setzt gute anatomische Kenntnisse der Nervenverläufe und das Bewusstsein der Möglichkeit einer Nervenverletzung voraus, und dennoch ist deren Auftreten trotz Vorsichtsmaßnahmen nicht immer vermeidbar.

In $\bullet$ Tab. 1 sind häufige Ursachen und die betroffenen Nerven zusammengestellt.

Auch Monate nach einem Unfallereignis können durch eine posttraumatische Arthrose oder bei Narbenbildungen im Gewebe sekundäre Nervenschädigungen entstehen [2]. Insgesamt sind Nervenläsionen zwar eher selten, haben aber, wenn sie auftreten, für den Betroffenen häu-

\title{
Traumatische Nervenläsionen
}

\section{Diagnose und Therapieentscheidungen}

fig eine erhebliche, lang anhaltende oder dauerhafte Funktionseinschränkung zur Folge, die mit Einschränkungen im Alltag und auch im Berufsleben einhergeht.

Entscheidend für die Prognose ist die zeitnahe Einleitung der neurologischen Diagnostik zur Feststellung des Läsionsortes und des Schweregrades, um frühzeitig die therapeutischen Schritte einzuleiten. Dies erfolgt im Zusammenspiel von klinisch neurologischer Untersuchung, elektrophysiologischer Zusatzdiagnostik und auch bildgebenden Verfahren und in engmaschiger Abstimmung mit weiteren involvierten Fachrichtungen wie Handchirurgie, Unfallchirurgie oder Neurochirurgie.

Bezüglich der Bildgebung gewinnt die Nervensonographie durch die Entwicklung von hochlinearen Breitbandschallköpfen (z. B. 5-18 MHz) und verbesserter Fähigkeit zur Bildverarbeitung zur Beurteilung der oberflächlich verlaufenden peripheren Nerven zunehmend an Bedeutung [3]. Der Nerv kann im Verlauf in transversaler und axialer Darstellung beurteilt werden. Die Untersuchungstechnik setzt allerdings Erfahrung und gute anatomische Kenntnisse voraus. Sie kann zur Lokalisation des Läsionsortes und zur Ursachenabklärung beitragen, was die Elektrophysiologie nicht kann. Ursachen von Nervenkompressionen lassen sich darstellen wie auch Tumoren und Neurome. Die Nervenstruktur stellt sich bei den größeren Nerven (- Abb. 1) transversal in streifiger Echotextur und durch Drehen des Schallkopfes um $90^{\circ}$ axial in wabiger Struktur dar, dadurch dass Nervenfaszikel (echoarm) und das dazwischen liegende Perineurium (echoreich) zu Darstellung kommen. Insbesondere völlige Kontinuitätsunterbrechungen sollten frühzeitig er- kannt werden, da sie unbedingt operativ neurorekonstruktiv angegangen werden müssen $[1,4]$.

Bei Nervenläsionen mit erhaltener Kontinuität ist es wichtig, den Schweregrad zu bestimmen, da auch hochgradige axonale Nervenschädigungen häufiger und früher operativ neurorekonstruktiv behandelt werden. Dies war bislang schwierig, da die Elektrophysiologie nur unzureichend zwischen hochgradigen und leicht bis mäßiggradigen axonalen Nervenläsionen zu differenzieren helfen kann. Mit der Sonographie können Ursachen von Nervenläsionen (z. B. Kompression des Nervs durch Osteosynthesematerial), die ebenfalls operativ behandelt werden müssen, aufgeklärt werden [5]. Aber auch das Ausmaß der axonalen Schädigung kann unter Umständen bestimmt werden [6].

\section{Pathophysiologie}

Zum besseren Verständnis der Verletzungsmuster sind anatomische Kenntnisse hilfreich (• Abb. 2).

Die meisten peripheren Nerven enthalten gemischt motorische, sensible, nozizeptive und autonome Nervenfasern. Umgeben und eingebettet sind die Axone von Bindegewebe, das einen großen Anteil eines peripheren Nervs ausmacht. Die äußere Hüllstruktur, das Epineurium, setzt sich aus Bindegewebe mit Anteilen von kollagenösen und elastischen Fasern zusammen, ihm kommt eine Schutzfunktion der Axone zu. Ein gewisses Maß an Faserondulation gewährleistet die physiologische Dehnbarkeit des Nervs. So wird eine kurzfristige Nervenelongation bis $12 \%$ der Ruhelänge ohne funktionellen Schaden toleriert. Die Durchblutung wird 


\begin{tabular}{ll|l}
\hline Tab. 1 & Häufige Ursachen für traumatische Nervenläsionen \\
\hline Nerv & Verletzung/Eingriff \\
\hline N. accessorius & Lymphknoten-PE \\
\hline N. radialis & $\begin{array}{l}\text { Dislozierte Oberarmfraktur, Reposition, Osteosynthese } \\
\text { Oberarm }\end{array}$ \\
\hline N. interosseus posterior & Reposition, Osteosynthese Radiusköpfchenfrakturen \\
\hline R. superficialis n. radialis & $\begin{array}{l}\text { Osteosynthese, Spaltung 1. Sehnenfach } \\
\text { N. ulnaris }\end{array}$ & $\begin{array}{l}\text { Spickdraht Osteosynthese bei kindlichen Ellenbogenfrak- } \\
\text { turen }\end{array}$ \\
\hline N. cutaneus antebrachii medialis & $\begin{array}{l}\text { Dekompression N. ulnaris } \\
\text { N. medianus und seine Endäste }\end{array}$ & $\begin{array}{l}\text { Karpaltunneloperation, Versorgung suprakondylärer Hu- } \\
\text { merusfrakturen bei Kindern }\end{array}$ \\
\hline N. axillaris & Humeruskopfluxation \\
\hline N. ischiadicus & Hüft-TEP, Acetabulumfraktur, gluteale Injektion \\
\hline N. obturatorius & Beckenringfraktur \\
\hline N. femoralis & Operation Leistenregion, Hüft-TEP \\
\hline N. cutaneus femoris lateralis & Knochenspanentnahme, Leistenoperation \\
\hline N. saphenus sowie R. infrapatellaris & Knieeingriffe, Venenstripping \\
\hline N. peronaeus & Knieoperationen, Kompartmentsyndrom \\
\hline N. suralis & Osteosynthese Knöchelfrakturen \\
\hline$P E$ Probeexzision, TEP Totalendoprothese \\
\hline
\end{tabular}

jedoch schon ab $8 \%$ Elongation beeinträchtigt, da hier bereits der venöse Rückstrom blockiert ist [7].

Bei $16 \%$ Dehnung werden Ischämien hervorgerufen. Dem Epineurium haftet eine weitere, kapillarisierte äußere Gleitschicht an, die das Gleiten des Nervs zwischen den Gewebeschichten während physiologischer Bewegungen gewährleistet. Bei neurorekonstruktiven Eingriffen oder traumatischen Läsionen ist es wichtig, eine solche Verschieblichkeit wiederherzustellen, um zu verhindern, dass der Nerv im Narbengewebe fixiert wird. Das Perineurium stellt die nächste Schicht dar, das die Faszikel einhüllt. Perineurale Fibroblasten haben äußerst vielfältige Aufgaben, insbesondere nach Nervenläsionen für die neuromatöse Umwandlung, da sie die Bindegewebsreaktion initiieren und unterhalten können. Das Perineurium scheint eine wichtige Rolle für die Erhaltung des physiologischen Milieus der leitenden Elemente zu spielen und wird als wesentlicher Bestandteil einer Diffusionsbarriere bzw. Blut-NervenSchranke gesehen. Die nächste Bindegewebsschicht unterhalb des Perineuriums ist das Endoneurium. Dieses umgibt die myelinisierten und Gruppen von nicht myelinisierten Axonen.
Die Rolle des Endoneuriums bei Verletzungen und in der Rekonstruktion scheint weniger gut verstanden zu sein. Insgesamt kommt den bindegewebigen Hüllstrukturen große Bedeutung bei der Nervenregeneration zu. Sie können neben Nährstofftransport durch Veränderung ihrer Syntheseprodukte nach einer Läsion Abräumvorgänge und auch Axonaussprossung induzieren [8].

\section{Schweregrade einer Nerven- läsion und deren Korrelat in Elektrophysiologie und Sonographie}

Die Einteilung peripherer Nervenläsionen erfolgt traditionell nach Seddon in 3 und nach Sunderland in 5 Schweregrade und orientiert sich an den verletzten Nervenstrukturen ( $\bullet$ Tab. 2). Da dies entscheidend für die Prognose der Läsion ist, liegt es im diagnostischen Bemühen, eine Einordnung des Läsionsausmaßes, orientiert an diesen Klassifikationen, vorzunehmen. Häufig liegen Mischbilder verschiedener Läsionsformen nebeneinander vor, oder ein Nerv ist an mehreren Stellen im Verlauf beschädigt. Zur Vereinfachung sollen im Folgenden die Läsionsgrade, orientiert an den beiden Klassifikationen, dargestellt und in Beziehung zu den zu erwartenden elektrophysiologischen und sonographischen Befunden gebracht werden (hierzu s. auch $\bullet$ Tab. 2).

Die leichteste Form stellt die Neurapraxie (Seddon 1, Sunderland Grad 1) dar, meist durch einen umschriebenen stumpfen Druck mit Quetschung des Nervs an dieser Stelle verursacht. Die Axone und ihre Hüllstrukturen bleiben intakt, und die Myelinscheide ist fokal geschädigt, das Ausmaß der Schädigung ist abhängig vom Ausmaß der Druckeinwirkung. Der klinische Befund reicht von kurzzeitigen Funktionsstörungen bis zum völligen segmentalen Markscheidenzerfall bei schwerer Nervenkompression [10]. Die Konsequenz ist ein Funktionsausfall des Nervs distal der Läsionsstelle. Die Prognose ist gut, da nur die Myelinscheide an dieser Stelle regenerieren muss. Neurographisches Korrelat ist der sog. Leitungsblock im Bereich der Läsion mit Amplitudenabfall der sensiblen Nervenaktionspotenziale (NAP) und motorischen Muskelantwortpotenziale (MAP) in der Neurographie. Distal vom Läsionsort bleiben dahingegen MAP und NAP unverändert. Das Elektromyogramm (EMG) bleibt bei der isolierten Myelinscheidenläsion im Verlauf unauffällig, und die für eine axonale Läsion typische Denervationstätigkeit in Form von Fibrillationen und PSW (,positive sharp wave"), die in etwa 14 Tage nach erfolgter Läsion nachweisbar ist, bleibt hier aus. So ist auch bei hochgradigen neurologischen Ausfällen die Differenzierung zwischen axonaler Läsion und der prognostisch günstigeren Neurapraxie durch die Elektrophysiologie innerhalb von 14 Tagen sicher möglich. Sonographisch stellt sich der Nerv bis auf eine gelegentlich zu beobachtende Auftreibung im Läsionsareal unauffällig dar.

Bei stärkerer Gewalteinwirkung kommt es auch zur Beschädigung von Axonen, wobei die Hüllstrukturen intakt bleiben (Seddon 2, Sunderland Grad 2). Ist das Endoneurium mit betroffen, liegt nach Seddon eine Axonotmesis und nach Sunderland Grad 3 vor. Das Perineurium, in das die Faszikel eingebettet sind, ist noch intakt. Man spricht von partieller Axonotmesis. Vom Läsionsort ausgehend, setzt ein Axonzerfall, die Waller- 
Degeneration, ein, der bis zum Erfolgsorgan fortschreitet und innerhalb von 5 bis 10 Tagen abgeschlossen ist. Nach Abschluss der Waller-Degeneration und phagozytotischen Abräumprozessen bilden proliferierende Schwann-Zellen das Hanke-Büngner-Band, das für die Nervenregeneration relevant ist. $\mathrm{Da}$ die Hüllstrukturen (Endo- und Perineurium) nicht beschädigt sind, diese aber als Leitstruktur für die neu aussprossenden Axone dienen, ist die Prognose für die Wiederherstellung der Funktion günstig. Klinisch besteht bei frischer Läsion motorisch meist eine Restfunktion, und die sensiblen Funktionen in dem vom betroffenen Nerv versorgten Hautareal sind nicht vollständig aufgehoben, da nur ein Teil der Axone beschädigt ist. In der Neurographie sind die Amplituden der MAPs und NAPs distal der Läsionsstelle reduziert, graduell verschieden in Abhängigkeit vom Ausmaß des Axonverlustes. Mit der Wiederaussprossung und Remyelinisierung ist auch die Nervenleitgeschwindigkeit reduziert.

Die aussagefähigere elektrophysiologische Messmethode bei dieser Fragestellung ist allerdings das EMG [11]. Nur Nervenläsionen, die mit einer axonalen Läsion verbunden sind, führen in den betroffenen Muskeln innerhalb von ca. 14 bis 21 Tagen nach stattgehabter Schädigung zur typischen Denervationstätigkeit. Es handelt sich um kleine Potenziale typischer Konfiguration, die spontan ohne Willküranspannung rhythmisch in Serien auftreten und wohl ausgelöst sind durch vermehrte Synthese von sonst nur embryonal vorkommenden Acetylcholinuntereinheiten, die dann nicht nur im Bereich der motorischen Endplatte, sondern auch in der Zellmembran inkorporiert vorkommen. Es wird diskutiert, dass sie möglicherweise einen chemotaktischen Reiz für die aussprossenden Axone darstellen. Im zeitlichen Verlauf mit Wiederaussprossung der Axone sind Reinnervationspotenziale im EMG nachweisbar. Durch Auswahl der zu untersuchenden Muskeln kann auf ein Nervenversorgungsgebiet, ein Segment oder den Plexus als Lokalisation der Läsion rückgeschlossen werden. Die Muskeln sind dieser Untersuchung gut zugänglich, und mit entsprechenden Atlanten

Trauma Berufskrankh 2017 • 19 (Suppl 3):S340-S346

https://doi.org/10.1007/s10039-017-0319-x

c) Springer Medizin Verlag GmbH 2017

\section{S. Seddigh}

\section{Traumatische Nervenläsionen. Diagnose und Therapieentscheidungen}

\section{Zusammenfassung}

Periphere Nerven können aufgrund ihrer Nähe zu knöchernen Strukturen oder auch bei sehr oberflächlichem Verlauf bei einem Trauma oder im Rahmen operativer Eingriffe geschädigt werden. Motorische und sensible Defizite oder gelegentlich auch neuropathische Schmerzen sind die Folge. Entscheidend für die Prognose ist neben der Lokalisationsdiagnostik die Einschätzung des Schweregrades der Läsion, die sich an den Klassifikationen nach Seddon 1-3 und nach Sunderland Grad 1-5 orientiert und mithilfe der klinisch neurologischen Untersuchung, der Elektrophysiologie und bildgebenden Verfahren vorgenommen wird. Hier ist insbesondere die Nervensono- graphie als zunehmend interessante und aussagekräftige Methode zu erwähnen. Leicht bis mäßiggradige Nervenläsionen haben aufgrund der Regenerationsfähigkeit peripherer Nerven eine günstige Prognose, während höhergradige Nervenläsionen unter konservativen Therapiebedingungen häufig eine ungünstige Prognose haben, es kommt zu Defektheilungen. Komplette Nervendurchtrennungen müssen immer operativ behandelt werden.

\section{Schlüsselwörter}

Schweregrad · Neurologische Untersuchung · Elektrophysiologie · Nervensonographie . Prognose

\section{Traumatic nerve injury. Diagnostic and treatment decisions}

\section{Abstract}

Peripheral nerves can be damaged due to their proximity to bony structures, to a very superficial course of trauma or during operative interventions. The sequelae are motor and sensory deficits or also occasionally neuropathic pain. Apart from the localization diagnostics, estimation of the severity of the lesion is decisive for the prognosis. This is oriented to the classification systems of Seddon 1-3 and Sunderland grades 1-5 and is undertaken with the help of neurological examination, electrophysiology and imaging procedures. Nerve sonography must be particularly mentioned as an increasingly more interesting and informative method. Light to moderate grade lesions have a favorable prognosis due to the regeneration capabilities of peripheral nerves, whereas higher grade nerve lesions often have an unfavorable prognosis under conservative treatment conditions resulting in defective healing. Complete nerve severance must always be treated surgically.

\section{Keywords}

Severity · Neurological examination . Electrophysiology · Nerve sonography . Prognosis sind auch tiefer gelegene Muskeln sicher auffindbar. Je körpernäher die Läsion lokalisiert ist und sich somit in größerer Entfernung zum Zielorgan (Muskel) befindet, desto länger und störanfälliger ist der Reinnervationsprozess, wobei das Axonwachstum mit einer Geschwindigkeit von ca. $1 \mathrm{~mm} /$ Tag voranschreitet. Nervenfasern sprossen entweder ganz neu aus, oder benachbarte intakte Axone übernehmen denervierte Muskelfasern. Elektrophysiologisches Korrelat sind polyphasische und sehr kleine naszierende Willkürpotenziale. Diese Reinnervationspotenziale sind in den dem Läsionsort am nächsten gelegenen Muskeln zu- erst nachweisbar. Regelmäßige Verlaufsuntersuchungen bieten wichtige Informationen zur Prognose.

Bei stärkerer oder bei spitzer Gewalteinwirkung mit noch hochgradigerer Nervenschädigung kommt es zusätzlich zu einer Kontinuitätsunterbrechung des Perineuriums (Sunderland Grad 4), man spricht von einer kompletten Axonotmesis. Die Binnenstruktur des Nervs ist zerstört oder narbig umgewandelt, und die Prognose der Reinnervationsvorgänge ist deutlich schlechter. Eine Abgrenzung der höhergradigen Axonotmesis mit zusätzlicher Schädigung der Hüllstrukturen (Sunderland Grad 3 und 

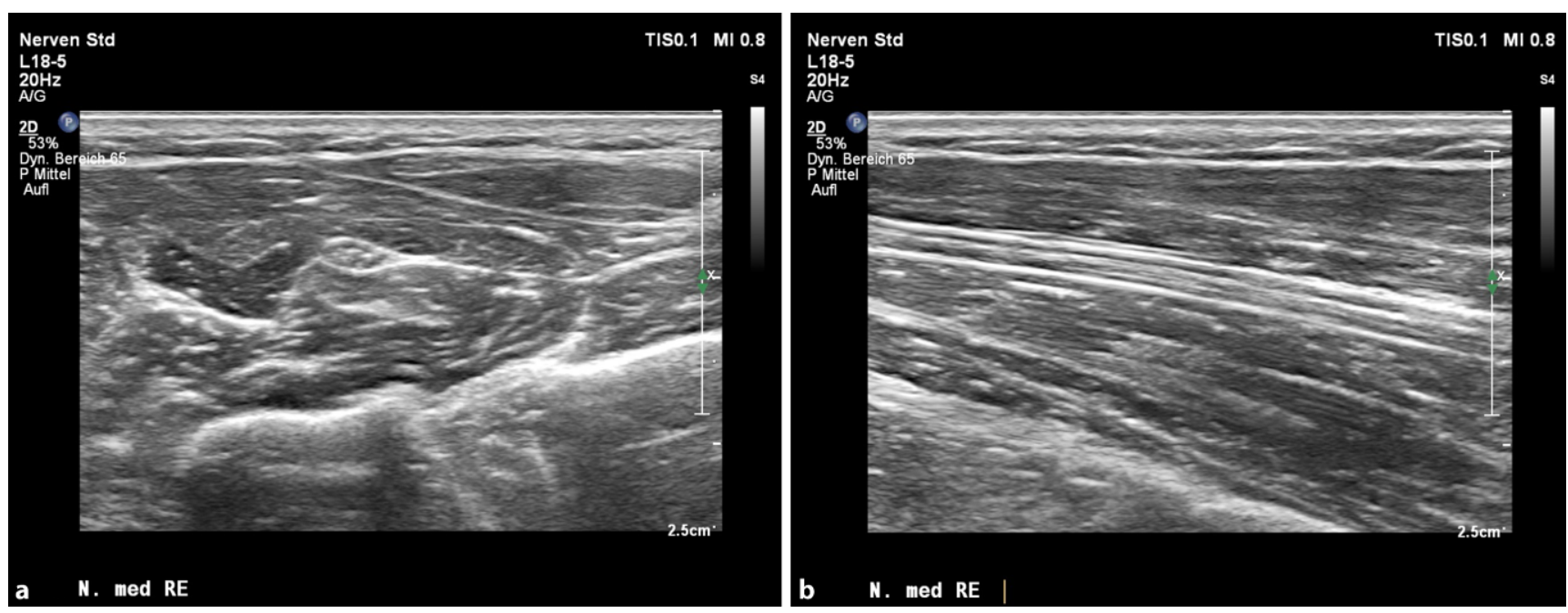

Abb. 1 - Sonographie des N. medianus a axial, b transversal

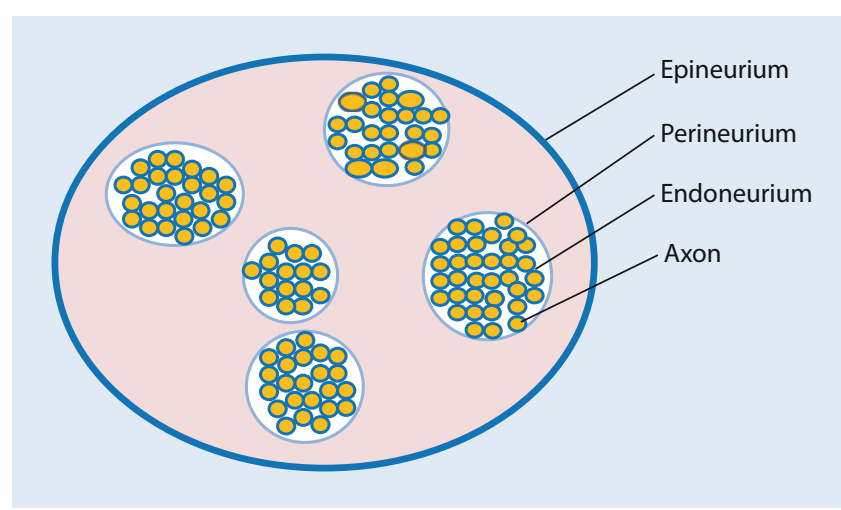

Abb. $2 \Delta$ Aufbau eines peripheren Nervs mit den unterschiedlichen Hüllstrukturen im Querschnitt

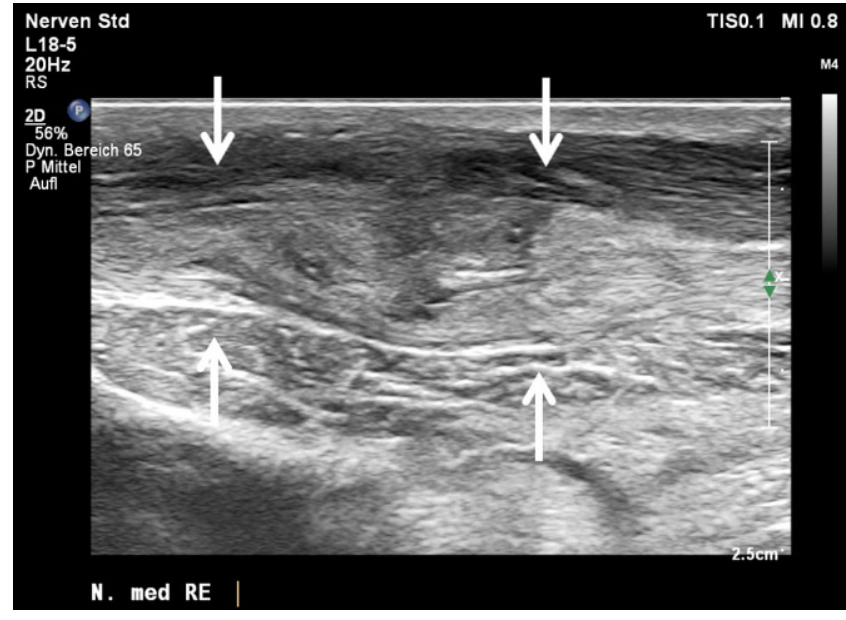

Abb. 3 × Kontinuitätsneurom nach N.-medianus-Läsion
4) gelingt elektrophysiologisch nicht, wie auch eine Abgrenzung zu einer völligen Kontinuitätsunterbrechung (Neurotmesis) elektrophysiologisch nicht sicher möglich ist. Ein Ausbleiben von Reinnervationspotenzialen im EMG nach 3 bis 6 Monaten ist prognostisch als ungünstig zu bewerten. Ist die zu überwindende Distanz von der Läsion bis zum Erfolgsorgan aber sehr groß (z. B. proximale N.-radialis-Läsion oder N.-ischiadicus-Läsion), kann dies auch noch mehr Zeit in Anspruch nehmen. Sonographisch kann eine Differenzierung zwischen Sunderland Grad 3 mit intaktem Perineurium und Sunderland Grad 4 mit beschädigtem Perineurium unter Umständen gelingen. Bei hochgradigen Nervenläsionen mit Beschädigung des Perineuriums ist die typische
Echotextur aufgehoben, die Faszikel erscheinen aufgetrieben und echoarm, die Nervenquerschnittsfläche ist vergrößert [12]. Fehlende Leitstrukturen haben eine wenig zielgerichtete Axonaussprossung mit Knäuelbildung zur Folge. Es entstehen Kontinuitätsneurome (• Abb. 3), die sonographisch gut darstellbar sein können. Somit kann die Sonographie beitragen, zwischen partieller und vollständiger Axonotmesis zu differenzieren. Bei Letzterem, insbesondere wenn sich ein Kontinuitätsneurom nachweisen lässt, ist ein neurorekonstruktiver Eingriff $\mathrm{zu}$ favorisieren. Bei einer völligen Kontinuitätsunterbrechung, der Neurotmesis (Seddon 3, Sunderland 5), kann der geschädigte Nerv nicht ohne operative Intervention heilen. Klinisch kommt es distal der Läsion zum völ- ligen Verlust von Willküraktivität und vollständig aufgehobener Berührungsempfindung und Spitzdiskrimination. Elektrophysiologisch ist nach 14 bis 21 Tagen Denervationsaktivität nachweisbar. Sonographisch lässt sich ggf. die Kontinuitätsunterbrechung und im weiteren Verlauf evtl. auch ein Stumpfneurom darstellen.

Ziel ist es, möglichst früh Aufschluss über Schweregrad und Lokalisation der Läsion zu erlangen, um das therapeutische Procedere zu bahnen.

\section{Klinischer Untersuchungs- befund}

Dem klinisch neurologischen Befund mit der Erfassung des Muskelstatus und der sensorischen Ausfälle kommt im 
Tab. 2 Einteilung traumatischer Nervenläsionen [9]

Seddon (1942)

Sunderland (1951)

\section{Neurapraxie}

Läsion ohne Durchtrennung von Axonen

\section{Axonotmesis}

Partiell, Kontinuitätsunterbrechung von

Axonen bei erhaltenen Nervenhüllen

Komplett nur das Epineurium ist noch intakt

Neurotmesis

Komplette Durchtrennung des Nervs mit Dehiszenz

Grad 1: Blockade der Nervenleitung ohne Axonverletzung

Grad 2: Axonunterbrechung mit intaktem Endoneurium

Grad 3: mit Läsion des Endoneuriums, Perineurium bzw. BM der Schwann-Zellen sind intakt

Grad 4: mit Läsion des Perineuriums, Kontinuität des Nervs ist äußerlich noch erhalten $(4 a / b)$

Grad 5: komplette Durchtrennung des Nervs mit Dehiszenz

BM Basalmembran

\section{Tab. 3 Klinischer Befund, Elektrophysiologie und Sonographie bei Nervenläsionen}

\begin{tabular}{|c|c|c|c|c|}
\hline Läsionsart & $\begin{array}{l}\text { Elektromyo- } \\
\text { graphie }\end{array}$ & Neurographie & Klinik & Sonographie \\
\hline \multicolumn{5}{|l|}{ Neurapraxie } \\
\hline Sunderland I & $\begin{array}{l}\text { Direkt: normal } \\
4-14 \mathrm{~T} \text { : normal } \\
\text { >14 T: normal }\end{array}$ & $\begin{array}{l}\text { Leitungsblock, } \\
\text { distal der Läsion } \\
\text { normal }\end{array}$ & $\begin{array}{l}\text { Parese, Restfunk- } \\
\text { tion erhalten } \\
\text { Hypästhesie/ } \\
\text { Hypalgesie } \\
\text { Vegetativ: nor- } \\
\text { mal }\end{array}$ & $\begin{array}{l}\text { Keine Veränderun- } \\
\text { gen/leichte echoar- } \\
\text { me Schwellung }\end{array}$ \\
\hline \multicolumn{5}{|l|}{ Axonotmesis } \\
\hline $\begin{array}{l}\text { Partiell: } \\
\text { Sunderland II/III }\end{array}$ & $\begin{array}{l}\text { Direkt: etwas } \\
\text { Willkür } \\
14 \text { T: PSA, } \\
\text { etwas Willkür } \\
3 \text { M: PSA, po- } \\
\text { lyphasische } \\
\text { WP } \\
\text { 6-9 M: (PSA), } \\
\text { überhöhte WP }\end{array}$ & $\begin{array}{l}\text { Direkt: normal } \\
8 \mathrm{~T}: \text { Amplituden- } \\
\text { reduktion des } \\
\text { MAP } \\
3 \text { M: Amplitu- } \\
\text { denreduktion } \\
\text { des MAP }\end{array}$ & $\begin{array}{l}\text { Parese, Restfunk- } \\
\text { tion erhalten } \\
\text { Hypästhesie/ } \\
\text { Hypalgesie } \\
\text { Vegetativ: nor- } \\
\text { mal }\end{array}$ & $\begin{array}{l}\text { Vergrößerung Ner- } \\
\text { venquerschnittsflä- } \\
\text { che } \\
\text { Echoarme Auftrei- } \\
\text { bung einzelner Faszi- } \\
\text { kel } \\
\text { Echotextur evtl. } \\
\text { gestört }\end{array}$ \\
\hline $\begin{array}{l}\text { (Sub)total: } \\
\text { Sunderland IV }\end{array}$ & $\begin{array}{l}\text { Direkt: etwas } \\
\text { Willkür } \\
14 \text { T: PSA, kein/ } \\
\text { einzelne WP } \\
3 \text { M: PSA, kein/ } \\
\text { einzelne WP } \\
\text { 6-9 M: (PSA), } \\
\text { evtl. polyphasi- } \\
\text { sches WP }\end{array}$ & $\begin{array}{l}\text { Direkt: normal } \\
8 \mathrm{~T}: \text { fehlendes } \\
\text { MAP } \\
3 \text { M: fehlendes } \\
\text { MAP }\end{array}$ & $\begin{array}{l}\text { Plegie (KG 0) } \\
\text { Anästhesie/ } \\
\text { Analgesie } \\
\text { Trophische Stö- } \\
\text { rungen }\end{array}$ & $\begin{array}{l}\text { Vergrößerte Nerven- } \\
\text { querschnittsfläche } \\
\text { Echoarme Auftrei- } \\
\text { bung der Faszikel } \\
\text { Verminderte Dar- } \\
\text { stellbarkeit der fas- } \\
\text { zikulären Echotextur } \\
\text { (ggf. Kontinuitäts- } \\
\text { neurom) }\end{array}$ \\
\hline \multicolumn{5}{|l|}{ Neurotmesis } \\
\hline Sunderland V & $\begin{array}{l}\text { Direkt: keine } \\
\text { Willkür } \\
\text { >14 T: PSA, } \\
\text { keine Willkür } \\
3 \text { M: PSA, keine } \\
\text { Willkür } \\
\text { 6-9 M: weniger } \\
\text { PSA, keine } \\
\text { Willkür }\end{array}$ & $\begin{array}{l}\text { Direkt: normal } \\
8 \mathrm{~T}: \text { fehlendes } \\
\text { MAP } \\
3 \text { M: fehlendes } \\
\text { MAP }\end{array}$ & $\begin{array}{l}\text { Plegie (KG 0) } \\
\text { Anästhesie/ } \\
\text { Analgesie } \\
\text { Trophische Stö- } \\
\text { rungen }\end{array}$ & $\begin{array}{l}\text { Kontinuitätsunter- } \\
\text { brechung des epi- } \\
\text { faszikulären Epineu- } \\
\text { riums partiell oder } \\
\text { komplett } \\
\text { Ggf. Nachweis eines } \\
\text { Stumpfneuroms }\end{array}$ \\
\hline
\end{tabular}

Hinblick auf die Lokalisation der Läsion eine besondere Bedeutung zu. Unter Kenntnis der Neuroanatomie kann auf ein Nervenversorgungsgebiet, ein Segment oder den Plexus als Lokalisation der Läsion rückgeschlossen werden. Das Verteilungsmuster der Paresen gibt auch Aufschluss über die Läsionshöhe innerhalb eines Nervs, da nur Muskeln, deren Nervenfasern distal der Läsionsstelle abgehen, betroffen seien können. Der Schweregrad der Lähmungen wird nach den Kraftgraden (0-5) beschrieben. Der Muskeltonus ist reduziert, was direkt nach einer Schädigung im Seitenvergleich sichtbar ist. Innerhalb von 1 bis 2 Wochen wird auch eine Muskelatrophie sichtbar. Bei reduzierter, aber erhaltener Restkraft des Muskels ist eine hochgradige Nervenläsion oder Kontinuitätsunterbrechung unwahrscheinlich. Ein Leitungsblock durch eine Neurapraxie kann aber durchaus eine hochgradige Parese bedingen. Hier trägt aber die Elektrophysiologie entscheidend zur Klärung bei.

Die Sensibilität ist im Versorgungsgebiet eines Nervs auf der Haut bezüglich der Qualitäten Berührung, Spitz-StumpfDiskrimination und Empfindung thermischer Reize reduziert oder bei hochgradigen Läsionen ganz aufgehoben. Bis auf wenige Ausnahmen (N. suprascapularis, N. accessorius) hat jeder Nerv des Körpers ein ihm exklusives Versorgungsareal, in dem die sensiblen Defizite nachweisbar sind. In diesem Areal befinden sich in der Regel auch trophische Störungen, das Schwitzen und die Hautbeschaffenheit. Größere Variabilität gibt es bei Plexusläsionen. Radikuläre Syndrome haben einen segmentalen, häufig diskreteren Sensibilitätsausfall aufgrund einer größeren Überlappung der Versorgungsgebiete benachbarter Segmente.

\section{Schlussfolgerung}

Durch die Kombination klinisch neurologischer, elektrophysiologischer und sonographischer Verlaufsuntersuchungen sollten früh Aussagen zur Lokalisation und Schwere einer Nervenverletzung vorgenommen werden. Die Einschätzung der Läsionsschwere orientiert sich an der Klassifikation nach Seddon und 


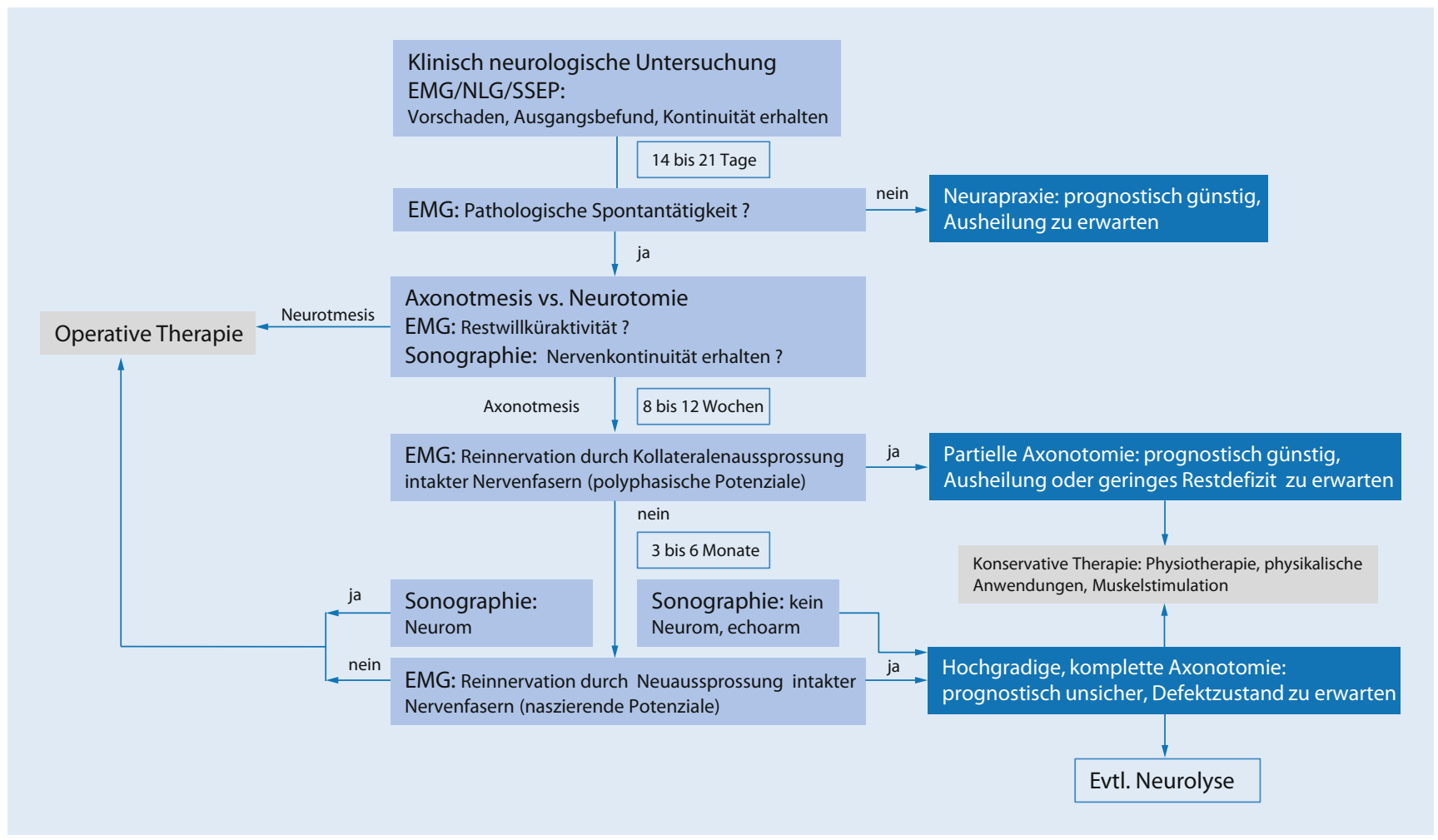

Abb. $4 \Delta$ Diagnostisches und therapeutisches Vorgehen bei Nervenläsionen. EMG Elektromyogramm, NLG Neurographie,

SSEP Somatosensibel evozierte Potentiale

Sunderland, wobei sich die Einschätzung der Befundkonstellation an den EMGund Sonographiebefunden orientiert (• Tab. 3).

Bei weniger schwerwiegenden Läsionen nach Sunderland Grad 1-3, ist ein konservatives Vorgehen anzustreben. Durch regelmäßige Verlaufsuntersuchungen mit dem EMG können Aussagen zum Stand des Reinnervationsprozesses und damit zur Prognose getroffen werden. Unterstützend finden Physiotherapie, physikalische Therapie sowie elektrische Muskelstimulation statt [13].

Der Nachweis einer Neurotomie macht regelmäßig ein operatives Vorgehen zur Nervenrekonstruktion nötig. Auch bei der kompletten Axonotmesis, sofern sich die Diagnose sichern lässt, wird zunehmend ein frühzeitiges operatives Vorgehen angestrebt, insbesondere wenn sich ein Kontinuitätsneurom nachweisen lässt.

Ein Vorschlag zum zeitlichen Ablauf und eine Entscheidungshilfe zum Procedere im zeitlichen Ablauf nach erfolgter Nervenschädigung sind in • Abb. 4 dargestellt. Insgesamt ist ein interdisziplinär eng abgestimmtes Vorgehen mit direktem Informationsaustausch besonders hilfreich in der Abstimmung des therapeutischen Procedere, insbesondere bei unklaren oder schwer interpretierbaren Befundkonstellationen.

\section{Fazit für die Praxis}

- Eine Nervenläsion sollte frühzeitig durch einen Neurologen klinisch und elektrophysiologisch untersucht werden.

- Komplizierte Nervenläsionen sollten in interdisziplinärer Kooperation behandelt werden.

- Elektrophysiologische Untersuchungen helfen innerhalb der ersten 3 Wochen, axonale Nervenläsionen von der prognostisch günstigen Neurapraxie abzugrenzen. Sie dienen durch Beurteilung der Reinnervationsaktivität im weiteren Verlauf der Prognoseeinschätzung.

- Die Nervensonographie gewinnt zunehmend an Bedeutung in der Beurteilung peripherer Nerven. Sie kann hilfreich in der Differenzierung von kompletter und partieller Axonotmesis sein und zur Ursachenabklärung einer Läsion beitragen.

- Leicht- bis mäßiggradige Nervenläsionen sind die Domäne der konservativen Therapie. Hochgradige Nervenläsionen sollten bei Nachweis eines Kontinuitätsneuroms oder völligem Ausbleiben der Reinnervation auch operativ behandelt werden. Eine vollständige Nervendurchtrennung wird operativ behandelt.

\section{Korrespondenzadresse}

Dr. S. Seddigh

BG-Klinikum Duisburg

Großenbaumer Allee 250, 47249 Duisburg,

Deutschland

susann.seddigh@bg-klinikum-duisburg.de

\section{Einhaltung ethischer Richtlinien}

Interessenkonflikt. S. Seddigh gibt an, dass kein Interessenkonflikt besteht. 


\section{Übersicht}

Dieser Beitrag beinhaltet keine vom Autor durchgeführten Studien an Menschen oder Tieren.

The supplement containing this article is not sponsored by industry.

\section{Literatur}

1. Antoniadis $G$, Kretschmer T, Pedro MT et al (2014) latrogene Nervenläsionen. Dtsch Arztebl 16:273-279

2. Bickert B (2014) Nervenverletzungen an der Hand. Trauma Berufskrankh 16(Suppl 2):169-172

3. Krämer HH, Kele H (2016) Nerven - Muskelsonographie. In: Kaps M, von Reutern G, Stolz E et al (Hrsg) Sonographie in der Neurologie. Thieme Verlag, Stuttgart, S273-280

4. Glocker F, Egger K (2015) Ein Pro - so viel wie möglich Bildgebung. Klin Neurophysiol 46:90-92

5. Schelle T (2015) Was kann die Neurosonografie. Klin Neurophysiol 46:79-89

6. Gruber H (2013) Nervenultraschall - neue klinische Anwendungen: eine auch kritische Betrachtung. OERG News 04/2013. OERG, Wien

7. Wanschitz J (2013) Pathophysiologie von Nervenläsionen. Neurologisch (Wien) 2:1-3

8. Sulaiman W, Gordon T (2013) Neurobiology of peripheral nerve injury, regeneration, and functional recovery: from bench top research to bedside application. Ochsner J13:100-108

9. AWMF -Register Nr. 005/010 S3 Leitlinie Versorgung peripherer Nervenläsionen, Stand 3/16 $\mathrm{S}: 1-97$

10. Pöschel P, Schulte-Matler WJ (2012) Neurophysiologische Diagnsotik bei traumatischen Nervenläsionen. Klin Neurophysiol 43:1-9

11. Bischoff C, Pöschl P, Dreger J et al (2015) Kontra-und Bilduntersuchungen in der Diagnostik der Erkrankungen peripherer Nerven - nur in speziellen Ausnahmen sinnvoll und notwendig. Klin Neurophysiol 46:93-96

12. Peer S (2009) Ultraschall in der Diagnsotik peripherer Nervenläsionen. J Neurol Neurochir Psychitr 10(1):54-59

13. Ninkovic M (2013) latrogenen Nervenläsionen Therapiemöglichkeiten aus der Sicht der physikalischen Medizin und Rehabilitation. Neuro 2:1-5 